\title{
Majority Preference for Subsidies over Redistribution
}

\author{
David Austen-Smith \\ Departments of Political Science and of Economics \\ Northwestern University \\ Evanston IL 60208
}

This version, December 28th 2001 


\begin{abstract}
Among other activities, democratic governments redistribute resources directly through tax schemes that explicitly benefit the poor and indirectly through subsidizing particular goods and services that do not. Indeed, in some cases the effective redistribution under subsidy policies is clearly away from the poor. This paper studies when a majority might prefer subsidy policies over direct income redistribution in economies with mean greater than median income. The main result is a set of necessary and sufficient conditions for subsidies to be majority preferred to direct redistribution: in sum, subsidies are strictly majority preferred to redistribution when the gap between median and mean incomes is not "too great".
\end{abstract}




\section{Introduction}

In a relatively recent interview Sir Peter Hall (1999), former director of the Royal Shakespeare Company and the National Theatre in England, is quoted as saying that "No government will do it, because there are no votes in the arts, but I would love to see tickets for theatre and opera made ridiculously cheap". Even under the dubious presumption that all individuals have a strict preference to see theatre and opera, Hall's claim about the political economy of arts funding raises an interesting puzzle about why majoritarian societies often redistribute resources indirectly through subsidizing the production or consumption of particular goods and services (as in education and health care, along with the arts) rather than directly through a purely redistributional tax scheme. The reason this is a puzzle is, I conjecture, less because "there are no votes" in subsidizing goods like the arts, but because it seems there could be more votes in a less targeted support policy. And the reason why this might be so lies in the asymmetries in welfare redistribution induced by subsidy policies. In particular, since contemporary societies are typically associated with distributions of income having mean exceed median income, we expect majoritarian governments to adopt redistributive policies that benefit the relatively disadvantaged majority. At least prima facie, subsidy policies do not necessarily reflect this.

In a contribution to the public education literature, Fernandez and Rogerson (1995) study a dynamic model of educational investment to consider why it is that although the cost of education is subsidized through proportional income taxation, the net effect is often a redistribution of welfare from the poor to those with higher incomes. Their answer is that if education subsidies are allocated only to those who purchase schooling, and if subsidies are funded through general taxation with the tax rate chosen by majority rule, then higher tax rates yield larger gross subsidies but also widen the effective demand for education which might reduce the per consumer subsidy. There are thus conflicting incentives and they show that the more unequal is the income distribution the more likely it is that the middle and high income brackets benefit at the expense of the poor in any majority rule equilibrium.

Along with the small extant literature on cash vs in-kind redistribution, ${ }^{1}$ Fernandez and Rogerson do not, however, ask whether a majority would in fact choose to use the sort of consumption subsidy policy they consider when

\footnotetext{
${ }^{1}$ See, for example, Besley and Coate (1991) and references therein.
} 
an alternative support policy is feasible. In particular, given the concern is with credit constraints, a natural alternate approach is simply to redistribute income and, in light of the Fernandez and Rogerson (1995) result, there is a prima facie presumption that a majority would prefer such redistribution to a consumption subsidy policy. So in what follows, I ask why a subsidy policy would be used to offset any credit constraints rather than a policy of direct income redistribution. The main result (for the highly simplified setup studied) is a set of necessary and sufficient conditions for subsidies to be majority preferred to direct redistribution. In effect, subsidy policy is majority preferred to redistribution when mean income is not too much greater than median income, where "too much" is determined relative to other parameters such as the unsubsidized consumption price. Intuitively, although the median income does not define the majority preferred tax rate under the subsidy policy, the median is a net beneficiary whichever policy, consumption subsidy or redistribution, is implemented; when the difference between median and mean income is not too great, the median's net return on his tax payment is greater with consumption subsidies than with direct redistribution.

To provide the best case for Sir Peter Hall's conjecture above to be correct, the basic model and results (developed in the next section) presume that all individuals have separable preferences between income (endowment) and consumption of the good and, further, that all individuals derive identical gross utility from the good. Thus the only thing preventing $100 \%$ of the population consuming is that not everyone can afford the (unsubsidized) price. Consequently, both the consumption subsidy policy and the pure redistribution policy involve moves only around the Pareto frontier rather than moves away from or toward efficiency. As such, the main question studied in the paper might be deemed largely irrelevant from a strictly welfare economic perspective. But this is clearly not the case from a political economy perspective; moreover, as shown later, the main results continue to hold when the consumption subsidy policy can induce inefficient resource allocation. In particular, an inefficient subsidy policy can be majority preferred to an efficient pure redistribution policy. 


\section{Model and Results}

The basic model is a single period, continuous type version of Fernandez and Rogerson (1995). There is a continuum of individuals with population size one. Individuals are distinguished by income (endowment), $y \geq 0$. The distribution of income is $F$ with average and median incomes, $\bar{y}$ and $\hat{y}$ respectively; assume $\bar{y}>\hat{y}$. Assume further that $F$ is differentiable almost everywhere with quasi-concave density $f$ such that $\lim _{y \rightarrow 0} f(y)=0$ and $\lim _{y \rightarrow 0} f^{\prime}(y) \geq 0 .^{2}$ Any individual may consume at most one unit of a good and all individuals value the unit at $v>0$. Since individuals consume at most one unit of the good, there is no ambiguity in using $v$ to identify the good itself.

Absent any subsidies, unit price is $p_{0}>0$ and $v>p_{0}$. In principle, therefore, if indeed Sir Peter Hall's wish "to see tickets for theatre and opera made ridiculously cheap" were to be realized, $100 \%$ of the population here would consume these arts. But although all individuals would like to consume the good, individuals are presumed credit constrained: other things equal, only individuals whose income is at least the unit price can consume $v$. To avoid trivial cases, the following assumption is maintained throughout the paper

$$
\bar{y}>p_{0}>0 .
$$

Without subsidies, therefore, those with at least average income can afford to buy $v$ but those with at most the median income may well be unable to consume the good.

There is a proportional income tax $t, 0 \leq t \leq 1$, to be chosen by majority preference. Tax revenues may be used in many ways. I consider two of these: general redistribution and subsidizing consumption.

\subsection{Direct redistribution}

Suppose taxes are used to subsidize consumption through redistribution. Specifically, tax-revenues are redistributed as lump-sum payments, leaving it to individuals to choose whether to buy the good or to consume the income directly.

\footnotetext{
${ }^{2}$ The restriction that $\lim _{y \rightarrow 0} f(y)=0$ is only for expository purposes. The qualitative results go through if the limit is strictly positive and finite.
} 
Under the general redistribution policy all individuals pay a proportion $t$ of their income in taxes and receive a common supplement $t \bar{y}$ to their disposable income. Thus, if, after paying taxes, income $y$ is enough to buy $v$ and the individual chooses to do so, then his or her payoff is

$$
u(t ; y, 1)=y(1-t)+t \bar{y}+v-p_{0} ;
$$

otherwise, the payoff is

$$
u(t ; y, 0)=y(1-t)+t \bar{y} .
$$

Let $\tilde{y}_{r}(t)$ denote the income at which an individual can just afford $v$ post-tax under the redistributive policy,

$$
\tilde{y}_{r}(t)(1-t)+t \bar{y}=p_{0} .
$$

Assuming that income redistribution is constrained to be no greater than that permitting all individuals to consume the good, all individuals with income below the mean, whatever their preferences over $v$, strictly prefer the tax-rate that minimizes $p_{0}-t \bar{y}$. Since $p_{0}<\bar{y}$ here, this rate is $p_{0} / \bar{y}<1$ and $\tilde{y}_{r}\left(p_{0} / \bar{y}\right)=0$.

Suppose the tax-rate is chosen by majority preference and, for every income and tax-rate $t \in\left[0, p_{0} / \bar{y}\right]$, let

$$
u^{*}(t ; y) \equiv \max [u(t ; y, 1), u(t ; y, 0)] .
$$

A tax-rate $t$ is a majority winner if and only if there is no distinct tax-rate $t^{\prime} \in\left[0, p_{0} / \bar{y}\right]$ such that

$$
\int_{Y\left(t^{\prime}, t\right)} d F(y)>\frac{1}{2}
$$

where $Y\left(t^{\prime}, t\right)=\left\{y: u^{*}\left(t^{\prime} ; y\right)>u^{*}(t ; y)\right\}$.

With the assumption that mean exceeds median income, the earlier remark on preferences over taxes directly implies

Proposition 1 The unique majority preferred tax rate under the redistributive policy is $\tau_{r}=p_{0} / \bar{y}$.

Figure 1 illustrates the equilibrium.

Figure 1 here 
Corollary 1 In equilibrium under the redistributive policy, all individuals with $v>0$ consume $v$ and there is a net redistribution of welfare from those with greater than mean income to those with less than mean income.

This is the usual result: welfare is redistributed from the relatively rich to the relatively poor. Furthermore, it is not hard to see that the majority equilibrium here implements the maximal value of the Benthamite aggregate social utility

$$
\begin{aligned}
W & =\int_{0}^{p_{0}} y d F(y)+\int_{p_{0}}^{\infty}\left[y+v-p_{0}\right] d F(y) \\
& =\bar{y}+\left[1-F\left(p_{0}\right)\right]\left(v-p_{0}\right) .
\end{aligned}
$$

By linear preferences and $v>p_{0}, W$ is maximized when all members of society who value $v$ consume it and this is achievable by imposing any purely redistributive tax-rate $t^{U B} \in\left[p_{0} / \bar{y}, 1\right]$.

\subsection{Consumption subsidies}

Suppose tax revenues are used to subsidize consumption of $v$ through price and let $p(t) \geq 0$ be the subsidized price. If an individual buys a unit of the good, his or her payoff is

$$
u(t ; y, 1)=y(1-t)+v-p(t) ;
$$

otherwise, the payoff is

$$
u(t ; y, 0)=y(1-t) .
$$

There are a variety of ways to model how the consumption subsidy is implemented and I focus on the simplest, the one used in Fernandez and Rogerson (1995). This is to assume individuals continue to purchase the good at price $p_{0}$ but receive a refund, so the tax revenue is distributed evenly over the set of individuals who consume and yields

$$
p(t)=p_{0}-\frac{t \bar{y}}{1-F\left(\tilde{y}_{s}(t)\right)} \geq 0,
$$

where $\tilde{y}_{s}(t)$ is the income at which an individual can, after paying the tax, just afford to consume $v$ :

$$
\tilde{y}_{s}(t)(1-t) \equiv p(t) .
$$


Comparing (2) with (3) gives $\tilde{y}_{r}(t)>\tilde{y}_{s}(t)$ for all $0<t<p_{0} / \bar{y}$ such that $\tilde{y}_{s}(t)>0$. So, at any interior tax rate, fewer people can afford to attend under the redistribution policy than under the subsidy policy. This is to be expected since not all recipients of the transfer under the redistribution policy choose to consume $v$.

Under the redistributive policy, all individuals' most preferred tax-rates lie on the boundaries of the admissible set, $\left\{0, p_{0} / \bar{y}\right\}$. Induced preferences, $u^{*}(t ; y)$, under the subsidy policy, however, are more complicated.

Consider an individual with income $y$. First note that if $y<\tilde{y}_{s}(t)$, $u^{*}(t ; y)=u(t ; y, 0)$ and the individual's induced payoff is linearly decreasing in $t$. Let $t_{y}=\tilde{y}_{s}^{-1}(y)$; then

$$
\lim _{t \uparrow t_{y}} u(t ; y, 0)=0<u\left(t_{y} ; y, 1\right)=v .
$$

Therefore, $u^{*}(t ; y)$ has a jump discontinuity at $t_{y}$, the rate at which $y$ is just enough to permit consumption at price $p(t)$. To identify the structure of the individual's indirect utility conditional on consuming the good, $u(t ; y, 1)$, it is useful to begin with the marginal consumer, $\tilde{y}_{s}(t)$.

Let the per capita subsidy at tax rate $t$ be $\sigma(t)=t \bar{y} /\left[1-F\left(\tilde{y}_{s}(t)\right)\right]$. Using (3), it is straightforward to check $d \tilde{y}_{s} / d t<0$ and $\sigma^{\prime}(t) \equiv d \sigma(t) / d t>0$ on the interval $\left(0, p_{0} / \bar{y}\right) .{ }^{3}$ And under the assumptions on the density $f$, it can further be shown that $\lim _{t \rightarrow 0} \sigma^{\prime \prime}(t)<0$ and $\lim _{t \rightarrow p_{0} / \bar{y}} \sigma^{\prime \prime}(t) \geq 0$. Although $\sigma^{\prime \prime}(t)$ could in principle exhibit multiple changes in sign, I assume hereafter that the distribution $F$ is sufficiently well-behaved that $\sigma^{\prime \prime}(t)$ changes sign at most once on $\left[0, p_{0} / \bar{y}\right]{ }^{4}$ Given this assumption, then, the per capita subsidy function is strictly increasing in $t$, strictly concave on $(0, a)$ and strictly convex on $\left(a, p_{0} / \bar{y}\right)$, where $0<a \leq p_{0} / \bar{y}$.

Let $t(y)$ maximize $u(t ; y, 1)$. Using the properties of the subsidy function $\sigma(t)$ and the fact that $t(y)$ maximizes $u(t ; y, 1)$ if and only if it solves $\max _{t}[\sigma(t)-t y]$, we obtain the following properties of $u(t ; y, 1)$.

Lemma 1 There exist incomes $0<y^{L}<y^{l}<\bar{y}<y^{h}<\infty$ such that:

(1) for all $y \geq y^{h}, u(t ; y, 1)$ is strictly decreasing in $t$ with $t(y)=0$;

\footnotetext{
${ }^{3}$ Formal proofs for this and subsequent claims are relegated to Appendix A.

${ }^{4}$ This assumption can (tediously) be checked directly to hold for triangular and piecewise linear distributions. Unfortunately, I have been unable to find a simple sufficient condition on the primitives to insure it holds; however, the assumption does not seem unreasonable.
} 
(2) for all $y \in\left[\bar{y}, y^{h}\right), u(t ; y, 1)$ is strictly quasi-concave with $t(y)$ interior and strictly decreasing in $y$;

(3) for all $y \in\left(y^{l}, \bar{y}\right), u(t ; y, 1)$ has a global maximum at $t(y)$ interior and a local maximum at $t=p_{0} / \bar{y}$; the interior maximum is strictly decreasing in $y$ with $\lim _{y \downarrow y^{l}} t(y)=t\left(y^{l}\right)<p_{0} / \bar{y}$;

(4) $u\left(t\left(y^{l}\right) ; y^{l}, 1\right)=u\left(p_{0} / \bar{y} ; y^{l}, 1\right)>u\left(t ; y^{l}, 1\right)$ for all $t \notin\left\{t\left(y^{l}\right), p_{0} / \bar{y}\right\}$;

(5) for all $y \in\left[y^{L}, y^{l},\right), u(t ; y, 1)$ has a global maximum at $t(y)=p_{0} / \bar{y}$ and an interior local maximum;

(6) for all $y<y^{L}, u(t ; y, 1)$ is strictly increasing in $t$ with $t(y)=p_{0} / \bar{y}$;

(7) for all $y, u^{*}(t ; y) \geq v$ at some $t$ implies $u^{*}\left(t^{\prime} ; y\right) \geq v$ for all $t^{\prime}>t$.

It is immediate from the lemma that there exists a unique global maximizer $t(y)$ for all $y \neq y^{l}$ and, for all $t \in\left(0, t\left(y^{l}\right)\right)$, the inverse mapping $t^{-1}(t)$ is a well-defined function. Hereafter, therefore, the notation $t(y)$ is understood to refer exclusively to the unique global maximizer for income $y$, ignoring the zero-measure event $\left\{y=y^{l}\right\}$. $^{5}$

Lemma 1 is illustrated in Figures 2 and 3 . Figure 2 shows $u^{*}(t ; y)$ for some $y \in\left(y^{l}, \bar{y}\right)$; Figure 3 describes both the globally most preferred tax rate as a function of income (conditional on consuming $v$ ) and the mapping $\tilde{y}_{s}(t)$.

Figures 2 and 3 here

Note that, by $(3)$ and $d \tilde{y}_{s}(t) / d t<0, \sigma^{\prime}(t)=\tilde{y}_{s}(t)-(1-t)\left[d \tilde{y}_{s}(t) / d t\right]>0$. Consequently, if $t$ is an interior utility maximizer for income $t^{-1}(t)=y$, then, as illustrated in Figure 3, $y>\tilde{y}_{s}(t)$ (and, evidently, if $y \geq y^{h}$, then $\left.\tilde{y}_{s}(t(y))=p_{0}<y\right)$.

Existence of majority winners in a model of price subsidies is, as observed by Fernandez and Rogerson (1995), complicated by individuals' induced preferences over tax-rates being non-single peaked. Indeed, as Lemma 1 suggests and Figure 2 illustrates, $u^{*}(t ; y)$ is neither single peaked nor continuous for any $y<p_{0}$, and it is easy to construct profiles $\left(u^{*}(\cdot ; y)\right)_{y}$ that fail order-restriction (Rothstein, 1990; Gans and Smart, 1996). The next result, however, provides necessary and sufficient conditions for there to exist a majority winner under the consumption subsidy scheme. For expository reasons, it is convenient to state the result for the case in which the median income's

\footnotetext{
${ }^{5}$ If $\lim _{y \rightarrow 0} f(y)>0$, then the lower bound on the set of strictly quasi-concave utilities with $t(y)<p_{0} / \bar{y}$ is smaller than $\bar{y}$. However, so long as the limit is finite, there must be an interior interval of types $\left(y^{l}, y\right), y<\bar{y}$, without quasi-concave utilities.
} 
most preferred tax rate is interior (i.e. $\hat{y}>y^{l}$ ), leaving the boundary case for subsequent discussion.

For any tax-rate $t^{\prime}<t(\hat{y})$, let $P\left(t^{\prime}\right)$ denote the set of more preferred alternatives for the median income individual:

$$
P\left(t^{\prime}\right)=\left\{t \in\left(t^{\prime}, p_{0} / \bar{y}\right]: u^{*}(t ; \hat{y})>u^{*}\left(t^{\prime} ; \hat{y}\right)\right\}
$$

Note that Lemma $1(3)$ and $\hat{y} \in\left(y^{l}, \bar{y}\right)$ imply $P\left(t^{\prime}\right)$ can be the union of two disjoint intervals although, given $t^{\prime}<t(\hat{y})$, if $t \in P\left(t^{\prime}\right)$ then $t^{\prime}<t$. For any two tax-rates $t^{\prime}<t^{\prime \prime}$, let $\omega\left(t^{\prime}, t^{\prime \prime}\right)$ be the income of an individual indifferent between $t^{\prime}$ and $t^{\prime \prime}$ (given $v>0$ ). By Lemma 1, $\omega\left(t^{\prime}, t^{\prime \prime}\right)$ is uniquely defined for any $t^{\prime}<t^{\prime \prime} .6$

Proposition 2 Suppose $t(\hat{y})<p_{0} / \bar{y}$. A tax-rate $\tau_{s} \in\left[0, p_{0} / \bar{y}\right]$ is a majority winner under the subsidy policy if and only if

(2.1) $\tau_{s} \in\left[\tilde{y}_{s}^{-1}(\hat{y}), t(\hat{y})\right)$;

(2.2) $\int_{\tilde{y}_{s}\left(\tau_{s}\right)}^{t^{-1}\left(\tau_{s}\right)} d F(y)=\frac{1}{2}$;

(2.3) $p_{0} / \bar{y} \notin P\left(\tau_{s}\right)$;

and, for all $t \in P\left(\tau_{s}\right)$,

(2.4) $\int_{\tilde{y}_{s}(t)}^{\omega\left(\tau_{s}, t\right)} d F(y)<\frac{1}{2}$.

Furthermore, if there exists a majority winner $\tau_{s}$, it is unique.

Condition (2.1) is fairly clear. If a tax-rate strictly exceeds the median's most preferred rate, $t(\hat{y})$, then more than half the population (at least all those with incomes $y \geq \hat{y}$ ) strictly prefer a smaller rate; and because the marginal consumer's income $\tilde{y}_{s}(t(\hat{y}))$ is not zero, more than half the population similarly prefer a lower tax to $t(\hat{y})$ itself. On the other hand, if the rate $t$ is strictly smaller than that just necessary to permit the median income individual from consuming the good, all those with incomes $y \leq \hat{y}$ strictly prefer a smaller rate since they do not consume at all at $t$.

Condition (2.2) says that if a tax-rate $t$ is a majority winner under the subsidy policy, then exactly $50 \%$ of the population must have incomes between that of the individual for whom $t$ is the most preferred rate and the

\footnotetext{
${ }^{6}$ Specifically, given $v>0$ and $t^{\prime}<\min \left\{t^{\prime \prime}, t(\hat{y})\right\}$,$$
\omega\left(t^{\prime}, t^{\prime \prime}\right)=\frac{\sigma\left(t^{\prime \prime}\right)-\sigma\left(t^{\prime}\right)}{t^{\prime \prime}-t^{\prime}} .
$$ 
marginal consumer's income, $\tilde{y}_{s}(t)$. Moreover, as the formal argument in Appendix A shows, if there are multiple such rates then only the maximal one satisfying (2.1) can be a majority winner. Given the condition, no incremental deviation from $t$ can be supported by a majority. Unfortunately, in view of Lemma 1 and the fact that $\tilde{y}_{s}(t)$ is decreasing in $t$, being a "local" majority winner, as assured by satisfaction of (2.1) and (2.2), is not enough.

Because all individuals strictly prefer to consume $v>0$ if they can pay the price and $\tilde{y}_{s}\left(p_{0} / \bar{y}\right)=0$, a majority of the population prefers the extreme tax-rate $p_{0} / \bar{y}$ to any rate $t<t(\hat{y})$ if the median does. And, by Lemma $1(3)$ and the assumption here that $y^{l}<\hat{y}$, the median has a local maximum at the boundary, $t=p_{0} / \bar{y}>t(\hat{y})$. Therefore, since condition (2.1) implies any potential majority winner $\tau_{s}$ must be strictly smaller than the median's most preferred rate, it is in principle possible for the median to prefer $p_{0} / \bar{y}$ to $\tau_{s}$ : condition (2.3) explicitly rules this out. And since the boundary is a local maximum, (2.3) also implies that no rate "close to" the boundary could be preferred by a majority to $\tau_{s}$.

Again because (2.1) implies $\tau_{s}<t(\hat{y})$, there remains the possibility that some rate in an interval $\left(\tau_{s}, t^{\prime}\right)$ containing $t(\hat{y})$ is preferred by a majority to $\tau_{s}$. To see that this can occur despite condition (2.2), consider a non-incremental increase in tax from $\tau_{s}$ to, say, $t$. Then there are more incomes $y$ for which $t>t(y)$ than there were for which $\tau_{s}>t(y)$ and, therefore, the proportion of the population in the upper half of the income distribution who prefer a smaller tax to $t$ goes up relative to the situation at $\tau_{s}$. On the other hand, the per capita subsidy increases with the tax-rate and $\tilde{y}_{s}(t)<\tilde{y}_{s}\left(\tau_{s}\right)$; hence the proportion of the population in the lower half of the income distribution who can now consume the good $v$ and who now prefer a yet higher rate similarly goes up. Which of these two countervailing effects dominates is obscure; condition (2.4), however, is equivalent to insuring the decrease in support for a higher rate among those already consuming at $\tau_{s}$ at least offsets the increase in support for such a rate among potential consumers at that higher rate.

As a characterization of equilibrium, Proposition 2 is a little disappointing. In particular, conditions (2.3) and (2.4) of the result amount to requiring directly that the maximal tax-rate satisfying conditions (2.1) and (2.2), $\tau_{s}$, can defeat any greater rate preferred to $\tau_{s}$ by the median income individual. Although the conditions can be stated explicitly in terms of the distribution $F$, doing so provides no clear characterization of the class of distributions 
for which they surely hold. ${ }^{7}$ On the other hand, given conditions (2.1) and (2.2), condition (2.3) is necessarily satisfied when the discrepancy between median and mean incomes is not too great, and condition (2.4) is typically satisfied when $\tau_{s}$ is "close" to the median's ideal point $t(\hat{y})$.

To check the first claim, note that condition (2.3) requires $u\left(\tau_{s} ; \hat{y}, 1\right) \geq$ $u\left(p_{0} / \bar{y} ; \hat{y}, 1\right)$ or, equivalently, that

$$
\left[1-F\left(\tilde{y}_{s}\left(\tau_{s}\right)\right)\right] \leq \frac{\tau_{s} \bar{y}^{2}}{p_{0}(\bar{y}-\hat{y})+\tau_{s} \bar{y} \hat{y}} .
$$

As $\hat{y}$ approaches $\bar{y}$ from below, the median's ideal tax-rate $t(\hat{y})$ similarly approaches the mean's ideal point $t(\bar{y})$ and, under the maintained assumptions, $t(\bar{y})>0$ for all $\hat{y}<\bar{y}$ and any admissible distribution of income. Therefore, by condition (2.1) of Proposition 2, the left side of the inequality approaches some limit strictly less than one as $\hat{y} \rightarrow \bar{y}$, whereas the right side goes to one. Consequently, condition (2.3) surely holds for $\hat{y}$ sufficiently close to $\bar{y}$ and the set $P\left(\tau_{s}\right)$ is an interval containing $t(\hat{y})$. To see the second claim, suppose $\tau_{s}$ is near the median's ideal point $t(\hat{y})$ and recall $\tau_{s}$ is necessarily the maximal tax-rate satisfying (2.1); then condition (2.2) and continuity of $F$ insures condition (2.4) obtains. (The next section develops an explicit numerical example illustrating both Proposition 2 and these remarks.)

The existence result states that if there is an interior majority preferred tax-rate, it is necessarily the most preferred tax rate of an individual with income strictly greater than the median. So, if the unsubsidized price, $p_{0}$, is sufficiently low relative to median income, such a majority-preferred rate could be zero. Since this possibility is essentially uninteresting, I assume hereafter that $p_{0}$ is sufficiently high to preclude the case. Then Figure 4 illustrates Proposition 2.

Figure 4 here

Proposition 2 says nothing about the boundary case, $t(\hat{y})=p_{0} / \bar{y}$. But, given the argument supporting Proposition 2, the answer is straightforward:

\footnotetext{
${ }^{7}$ For example, given (2.2) a sufficient (but not necessary) property for (2.4) is that, for all $t \in P\left(\tau_{s}\right), d\left[\int_{\tilde{y}_{s}(t)}^{\omega\left(\tau_{s}, t\right)} d F(y)\right] / d t \leq 0$; that is, for all $t \in P\left(\tau_{s}\right)$,

$$
\frac{d \tilde{y}_{s}(t) / d t}{d \omega\left(\tau_{s}, t\right) / d t} \leq \frac{f\left(\omega\left(\tau_{s}, t\right)\right)}{f\left(\tilde{y}_{s}(t)\right)} .
$$

Disentangling this inequality seems to offer no further insight beyond that in the text, below.
} 
if $\hat{y} \leq y^{l}$, there can be at most one majority winner and it is the maximal rate $p_{0} / \bar{y}$.

Because both the rich and poor prefer lower tax rates than that supported under majority preference, we immediately have the following implication of Proposition 2.

Corollary 2 If there exists an interior majority winner, $\tau_{s}<p_{0} / \bar{y}$, not all individuals consume the good $v$ under the consumption subsidy policy. There is a net redistribution of welfare from those with incomes $y \notin\left[\tilde{y}_{s}\left(\tau_{s}\right), t^{-1}\left(\tau_{s}\right)\right]$ in favour of those with incomes $y \in\left[\tilde{y}_{s}\left(\tau_{s}\right), t^{-1}\left(\tau_{s}\right)\right]$.

The poorest members of the community do not consume the good although they pay taxes, while the richest members consume the good but pay more in taxes than they gain through the subsidy. Hence, the net redistribution of income and welfare is from the rich and the poor to the middle income group.

Before going on to consider majority decisions over a direct redistribution policy, it is useful to consider which consumption subsidy policy would be chosen by a (constrained) Benthamite social welfare planner. Assuming the planner must adopt the consumption scheme studied here but is free to choose any proportional tax-rate to fund the scheme, she solves

$$
\begin{aligned}
\max _{t} W(t) & =\int_{0}^{\tilde{y}_{s}(t)} y(1-t) d F(y)+\int_{\tilde{y}_{s}(t)}^{\infty}[y(1-t)+v-p(t)] d F(y) \\
& =(1-t) \bar{y}+\left[1-F\left(\tilde{y}_{s}(t)\right)\right](v-p(t)) \\
& =(1-t) \bar{y}+\left[1-F\left(\tilde{y}_{s}(t)\right)\right]\left(v-p_{0}+\sigma(t)\right) \\
& =\bar{y}+\left[1-F\left(\tilde{y}_{s}(t)\right)\right]\left(v-p_{0}\right) .
\end{aligned}
$$

Hence,

$$
W^{\prime}(t)=-\left(v-p_{0}\right) f\left(\tilde{y}_{s}(t)\right) \frac{d \tilde{y}_{s}}{d t} .
$$

Therefore, since $d \tilde{y}_{s} / d t<0$ and $\lim _{y \downarrow 0} f(y)=0$, a Benthamite planner constrained to use the subsidy policy chooses the tax rate which induces all individuals to consume the good, $t^{C B}=p_{0} / \bar{y}$. Evidently, $\hat{y}>y^{l}$ implies $\tau_{s}<t^{C B}$.

In sum, recalling that an unconstrained Benthamite planner chooses the maximal tax rate, we have that whether or not a Benthamite social welfare 
maximizer is constrained by which sort of policy is feasible, he or she chooses an efficient tax-rate $t^{B}=p_{0} / \bar{y}$ and all individuals consume the good. This solution is implemented by majority preference under a direct redistribution policy but not, given $\hat{y}>y^{l}$, under a consumption subsidy policy. But Benthamite planners are hard to find. Suppose instead that society uses majority preference first to choose between using a subsidy policy or a direct redistribution policy and then, having made this choice, uses majority preference to determine the amount of tax-revenue to be raised by a proportional rate on income. Propositions 1 and 2 define the outcome to the second stage decisions and so, with all individuals sequentially rational, we have the following immediate consequence of those results.

Proposition 3 Assume a majority winner $\tau_{s}$ exists under the subsidy policy. Then a majority strictly prefers $\tau_{s}$ to the direct redistribution policy $\tau_{r}=p_{0} / \bar{y}$ if and only if $\tau_{s}<\tau_{r}$, and is otherwise indifferent.

In view of Proposition 2, Proposition 3 is obvious: the majority winner under the redistribution policy, $\tau_{r}$, is a feasible alternative under the subsidy policy; therefore, because existence of a majority winner under the subsidy policy, $\tau_{s}$, requires the median income individual to prefer (at least weakly) that outcome to any other, it must be the case that if $\tau_{s}<\tau_{r}$ then the subsidy policy must be preferred by a majority to direct redistribution. The intuition is that, although the net utility gain to the median under direct redistribution is positive, it is relatively small compared to that under consumption subsidies. In the latter case total consumption is lower and so the per capita subsidy for those able to afford the good is relatively high. Thus, while the nominal consumption price is higher with subsidies than with redistribution, the net return to the median's tax-bill under the subsidy policy is high relative to that under the redistribution policy.

\section{A Numerical Example}

Proposition 3 is not entirely satisfactory for the same reason that Proposition 2 is not entirely satisfactory: it is not a result directly on the primitives of the model, in particular, on the distribution of incomes $F$. To obtain a better intuition for the results, consider the following example. Although clearly somewhat contrived for computational reasons (for instance, the example violates the expository assumption that $\lim _{y \rightarrow 0} f(y)=0$ ), the example is far 
from pathological and is a reasonable approximation for a plausible class of unimodal distributions.

The distribution of income is assumed piecewise linear on the interval $[0,9]$ :

$$
F(y)=\left\{\begin{array}{cll}
\frac{y}{6} & \text { if } & 0 \leq y<1 \\
\frac{1}{6}+\frac{2(y-1)}{3} & \text { if } & 1 \leq y<2 \\
\frac{5}{6}+\frac{(y-2)}{42} & \text { if } & 2 \leq y \leq 9
\end{array}\right.
$$

In this case, the mean $\bar{y}=2$ and the median $\hat{y}=3 / 2$. Here, since $f(0)=1 / 6$, induced preferences over tax-rates for $y \geq 6 / 5$ are strictly quasi-concave; in particular, the median income person, and therefore a strict majority, has such induced preferences.

Even with a piecewise linear distribution of income, the function $\tilde{y}_{s}(t)$ is highly non-linear and this complicates the computations. Using the fact that the function has to be monotone on $[0,1]$ and that $\tilde{y}_{s} \in\left[0, p_{0}\right]$, it is easiest to solve out for the tax-rate $t$ as a function of the marginal consumer $\tilde{y}_{s}$, exploiting $\tilde{y}_{s}$ as the independent variable; details of the calculations supporting the numerical results to follow can be found in Appendix B, along with confirmation that the conditions of Proposition 2 hold.

Table 1 summarizes three cases, differing only in the price $p_{0} \cdot{ }^{8}$

\begin{tabular}{|c|c|c|c|c|c|}
\hline$p_{0}$ & $t(\hat{y})$ & $\tau_{s}$ & $t^{-1}\left(\tau_{s}\right)$ & $\tilde{y}_{s}\left(\tau_{s}\right)$ & $\tau_{r}$ \\
\hline \hline 1.67 & 0.66 & 0.60 & 1.69 & 0.75 & 0.83 \\
\hline 1.33 & 0.58 & 0.54 & 1.59 & 0.38 & 0.67 \\
\hline 1.00 & 0.50 & 0.50 & 1.50 & 0.00 & 0.50 \\
\hline
\end{tabular}

Table 1: Numerical examples

In the first row, the unit price of $v$ is $p_{0}=5 / 3$ and the median income individual $\hat{y}$ is unable to consume $v$ in the absence of some sort of subsidy or redistributive benefit (that is, $\tilde{y}_{s}^{-1}(\hat{y})>0$ ); the price $p_{0}$ is lower in the second row and the median can consume $v$ whether or not there are any subsidies. In both of these cases, however, the median's most preferred taxrate is strictly interior, $t(\hat{y})<\tau_{r}=p_{0} / \bar{y}$. Consequently, the majority winner under the subsidy policy, $\tau_{s}$, is likewise strictly below the majority preferred

\footnotetext{
${ }^{8}$ Throughout this section, numerical values are rounded to two decimal places.
} 
rate under the redistribution policy, $\tau_{r}$. In contrast, while the price in the final row is the lowest, the median's most preferred tax-rate is the boundary rate $\tau_{r}$, so $\tau_{s}=\tau_{r}=t(\hat{y})$ and all members of the polity consume the good. In sum, therefore, the lower is the initial price, the lower is the tax-rate required to induce consumption by all individuals and the more willing is a majority to support a direct redistribution policy.

Finally, suppose the maximal income is increased, holding $F(y)$ fixed as above for $y<2$ and keeping $F$ linear on $[2, \max y]$. Then $\hat{y}$ is invariant but $\bar{y}$ increases with $\max y$ and, for given price $p_{0}$, the majority winner under the subsidy scheme increases monotonically until the median individual's most preferred rate is on the boundary. For instance, for the case $p_{0}=$ $5 / 3$ and $\max y=12$, mean income $\bar{y}=2.25$ and the majority winner is (approximately) $\tau_{s}=0.62<t(\hat{y})=0.7$. Although I have been unable to prove such a comparative static generally, the result has a good intuition.

\section{$4 \quad$ Preference Heterogeneity ${ }^{9}$}

When all individuals share the same preference for the good, $v>0$, both the direct redistribution and the consumption subsidy policies are efficient. As such, the question of majority preference over the two might be considered irrelevant from the perspective of neoclassical welfare economics, which is primarily concerned with getting to the efficiency frontier and not with moves around it. Introducing the possibility of inefficiency complicates the analysis somewhat but leaves the qualitative results above essentially unaffected so long as the exent of the inefficiency is not too great. Specifically, assume not all individuals value the good $v$ at more than the market price $p_{0}$. A fixed proportion of the population $\phi>0$ value the good at $v^{\prime}<p_{0}$, with the remaining proportion $1-\phi$ continuing to value the good $v>p_{0}$. Assume that the distribution of basic preferences $v$ and $v^{\prime}$ for the good is independent of that for income and that $\phi$ is relatively small. Whereas the redistribution policy supports efficient resource allocation for all admissible tax-rates, any consumption subsidy such that $p(t)<v^{\prime}$ is economically inefficient. ${ }^{10}$

\footnotetext{
${ }^{9} \mathrm{I}$ am indebted to the referee, whose suggestion lead to this section. Of course, he or she can in no way be held responsible for what I have done with the suggestion.

${ }^{10} \mathrm{~A}$ type $v^{\prime}$ individual chooses to consume the good only if

$$
y(1-t)+v^{\prime}-p(t) \geq y(1-t) .
$$
}


The technical complication added by such preference heterogeneity is easily seen. Suppose $v^{\prime}>0$ and let $t^{\prime}$ be the tax-rate such that $p\left(t^{\prime}\right)=v^{\prime}$. Then the subsidy function $\sigma(t)$ drops discontinuously at $t^{\prime}$ : for all $t<t^{\prime}$, $\sigma(t)=t \bar{y} /\left[(1-\phi)\left(1-F\left(\tilde{y}_{s}(t)\right)\right)\right]$ and for all $t \geq t^{\prime}, \sigma(t)=t \bar{y} /\left[1-F\left(\tilde{y}_{s}(t)\right)\right]$. And this in turn induces a similar downward discontinuity in individuals' induced payoff functions over tax-rates.

Assuming $\phi$ is small, there are two cases that plainly leave the preceding analysis largely unaffected. The first case is when $v^{\prime}$ is close to $p_{0}$, so $t^{\prime}$ is close to zero and significantly smaller than the median's most preferred rate. Here, the conditions of Proposition 2 characterizing the majority winner $\tau_{s}$ are unaffected although any majority winner induces an inefficient allocation of resources (because almost a proportion $\phi$ of those consuming $p\left(\tau_{s}\right)$ have $v^{\prime}<$ $\left.p_{0}\right)$. The second case is when $v^{\prime}$ is close to zero, so $t^{\prime}$ is close to $\tau_{r}$ and greater than the median's most preferred rate. Under these circumstances there is no inefficient consumption at $\tau_{s}$ (because only those with $v>p_{0}$ consume at $p\left(\tau_{s}\right)$ ), but condition (2.2) and, possibly, condition (2.4) of Proposition 2 need some modification. In particular, condition (2.2) becomes

$$
\text { (2.2') }[1-\phi] \int_{\tilde{y}_{s}\left(\tau_{s}\right)}^{t^{-1}\left(\tau_{s}\right)} d F(y)=\frac{1}{2}
$$

and condition (2.4) becomes: for all $t \in P\left(\tau_{s}\right)$,

$$
\left(2.4^{\prime}\right)[1-\delta \phi] \int_{\tilde{y}_{s}(t)}^{\omega\left(\tau_{s}, t\right)} d F(y)<\frac{1}{2}
$$

where $\delta=1$ if $t<t^{\prime}$ and $\delta=0$ otherwise.

When $v^{\prime}$ is not "close to" either $p_{0}$ or zero, then the induced discontinuity at $t^{\prime}$ can complicate the existence of a majority winner further yet, with each possible case having to be considered separately. Doing this seems to add very little. It is, however, worth commenting on the polar case of $v^{\prime}=0$, where there is no inefficiency. In this circumstance, conditions (2.2) and (2.4) of Proposition 2 are replaced, respectively, by conditions (2.2') and (2.4') (with $\delta=1$ ); condition (2.3) is no longer necessary for a majority winner since it is possible for the median to prefer the extreme policy to $\tau_{s}$, but there to be fewer than a majority of the population with $v>0$ sharing the that

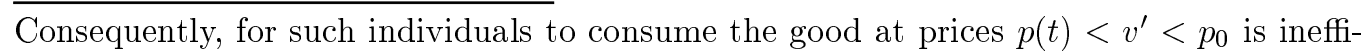
cient. 
preference. Given $\phi$ small, existence of a majority winner under the subsidy policy holds in the same settings as it does for $\phi=0$; more interesting is the observation that when such winners do exist, they are increasing in $\phi .{ }^{11}$ This makes perfectly good sense: as $\phi$ increases marginally, the per capita subsidy to those consuming the good $v>0$ also rises for any given tax rate $t>0$; therefore, for $\phi$ sufficiently small, if a majority supports a tax $\tau_{s}$ against the redistribution rate $\tau_{r}$ when $\phi=0$, then it can do so when $\phi$ increases a little. The point is illustrated using the numerical example above.

Consider the specification of the numerical example under which $p_{0}=$ $4 / 3$ and $y \in[0,9]$. When $\phi=0$, the majority winner is $\tau_{s} \approx 0.54$ with $\tilde{y}_{s}\left(\tau_{s}\right) \approx 0.38$. Suppose $\phi=0.05$. Then going through the calculations, taking account of the fact that not all the population chooses to consume at any price, there is a majority winner at $\tau_{s}^{\prime} \approx 0.58$ with $\tilde{y}_{s}\left(\tau_{s}^{\prime}\right) \approx 0.29$. Within some limits, therefore, the smaller the proportion of the population interested in consuming the good at all, the greater is the majority preferred general tax-rate to support that preference: in view of the quotation from Sir Peter Hall cited earlier, the result is of some interest.

\section{Conclusion}

It follows from Proposition 3 above that, pace Sir Peter Hall, even when there are potentially large numbers of votes in supporting consumption of some good like the arts, majoritarian incentives can lead to subsidies over redistribution at the expense of maximizing such consumption. Moreover, interior majority winners under the subsidy policy fail to maximize aggregate welfare (and might further be inefficient when not all individuals value the good as highly as the market price), whereas majority preference selects an aggregate welfare and consumption maximizing outcome under a direct redistribution policy. The model is extremely sparse. In particular, everyone values consumption of the good identically and there is no real production. While these assumptions can be found elsewhere it would be useful to ask what happens in a richer setting. On the other hand, the numerical example suggests the main result does not apply only to peculiar cases.

To conclude, it is worth commenting briefly on an alternative subsidy policy to that considered so far. ${ }^{12}$ Assume $v$ is produced under a zero profit

\footnotetext{
${ }^{11} \mathrm{~A}$ proof of this statement is in the Appendix.

${ }^{12}$ There are, of course, many alternative policies conceivable here. The focus on the
} 
condition and the tax revenue is used to lower the per unit sales price, say

$$
q(t)=\frac{p_{0}-t \bar{y}}{1-F\left(\tilde{y}_{q}(t)\right)}
$$

where $\tilde{y}_{q}(t)$ is defined by (3) with $q(t)$ replacing $p(t)$. Going through essentially the same reasoning as used above, it is straightforward to check that all of the qualitative results hold under this alternative specification. The one additional result worth noting is the following: existence of a majority preferred tax rate under the original subsidy policy, $\tau_{s}$, is a necessary condition for existence of a majority preferred tax rate under the alternative subsidy policy, but it is not sufficient. The reasons for this, as is a little tedious but straightforward to check, are that individuals' most preferred tax rates with $q(t)$ are less than those with $p(t)$, and that $q(t) \in\left(0, p_{0} / \bar{y}\right)$ implies $q(t)>p(t)$. Thus, appropriately applying Proposition 2 , the range of candidate tax rates for a majority winner with $q(t)$ is a proper subset of the range with $p(t)$.

two in the body of the paper is justified largely by empirical relevance and that, in many respects, they offer canonical alternatives with respect to redistribution. 


\section{Appendix A: Proofs}

Begin by confirming $d \tilde{y}_{s}(t) / d t<0$. Rewrite $(3)$ as

$$
\tilde{y}_{s}(t)(1-t)+\frac{t \bar{y}}{1-F\left(\tilde{y}_{s}\right)} \equiv p_{0} .
$$

Then $\tilde{y}_{s}(t)>0$ if and only if $t<p_{0} / \bar{y}$. Assuming this inequality holds, implicit differentiation through (4) gives

$$
\frac{d \tilde{y}_{s}}{d t}=\frac{\left[1-F\left(\tilde{y}_{s}\right)\right]\left[\left(1-F\left(\tilde{y}_{s}\right)\right) \tilde{y}_{s}-\bar{y}\right]}{(1-t)\left(1-F\left(\tilde{y}_{s}\right)\right)^{2}+t \bar{y} f\left(\tilde{y}_{s}\right)}<0
$$

with the inequality following from (1) and definition (3). Recall $\sigma(t)=$ $t \bar{y} /\left[1-F\left(\tilde{y}_{s}(t)\right)\right]$. Then

$$
\begin{aligned}
\sigma^{\prime}(t) & =\frac{\bar{y}}{\left[1-F\left(\tilde{y}_{s}\right)\right]^{2}}\left[1-F\left(\tilde{y}_{s}\right)+t f\left(\tilde{y}_{s}\right) \frac{d \tilde{y}_{s}}{d t}\right] \\
& =\frac{\bar{y}}{\left[1-F\left(\tilde{y}_{s}\right)\right]}+\sigma(t) h\left(\tilde{y}_{s}\right) \frac{d \tilde{y}_{s}}{d t}
\end{aligned}
$$

where $h(z) \equiv f(z) /[1-F(z)]$ is the hazard rate associated with the distribution of income. Substituting for $d \tilde{y}_{s} / d t$ and collecting terms confirms $\sigma^{\prime}(t)>0$ on $\left[0, p_{0} / \bar{y}\right]$. Differentiating a second time and collecting terms,

$$
\sigma^{\prime \prime}(t)=h\left(\tilde{y}_{s}\right)\left[\frac{d \tilde{y}_{s}}{d t}\left(\frac{\bar{y}}{\left[1-F\left(\tilde{y}_{s}\right)\right]}+\sigma^{\prime}(t)\right)+\sigma(t) \frac{d^{2} \tilde{y}_{s}}{d t^{2}}\right]+\sigma(t) h^{\prime}\left(\tilde{y}_{s}\right)\left(\frac{d \tilde{y}_{s}}{d t}\right)^{2}
$$

By assumption, $\lim _{y \rightarrow 0} f(y)=0$ and $\lim _{y \rightarrow 0} f^{\prime}(y) \geq 0$; therefore, $\lim _{t \rightarrow 0} \sigma^{\prime \prime}(t)<$ 0 and $\lim _{t \rightarrow p_{0} / \bar{y}} \sigma^{\prime \prime}(t) \geq 0$ as claimed. Hereafter, the derivative $\sigma^{\prime \prime}(t)$ is assumed to change sign (minus/plus) at most once on the domain $\left[0, p_{0} / \bar{y}\right]$.

Lemma 1 is an almost immediate consequence of the preceding properties of the subsidy function and the linearity of individuals' preferences in own income.

\section{Proof of Lemma 1.}

Using the identitity (4),

$$
\sigma(t) \equiv p_{0}-\tilde{y}_{s}(1-t)
$$


SO

$$
\frac{d \sigma}{d t} \equiv \tilde{y}_{s}-\frac{d \tilde{y}_{s}}{d t}(1-t)>0
$$

If $y$ is sufficient to purchase $v$, then the payoff is $u(t ; y, 1)$. Hence,

$$
\begin{aligned}
\left.\frac{d u}{d t}\right|_{t<p_{0} / \bar{y}} & =-y-p^{\prime}(t) \\
& =\sigma^{\prime}(t)-y \\
& =\left[\tilde{y}_{s}-y\right]-\frac{d \tilde{y}_{s}}{d t}(1-t) .
\end{aligned}
$$

By definition of $\tilde{y}_{s}$, if an individual with income $y$ consumes $v$ then $\left[\tilde{y}_{s}-\right.$ $y] \leq 0$. And by definition of $\tilde{y}_{s}$ and the assumption on $p_{0}, t=0$ implies $\tilde{y}_{s}=p_{0}<\bar{y}$. Consequently, $\left[d \tilde{y}_{s} / d t\right]_{t=0}=p_{0}-\left[\bar{y} /\left(1-F\left(p_{0}\right)\right)\right]$ implying

$$
\left.\frac{d u(t ; y, 1)}{d t}\right|_{t=0}=\frac{\bar{y}}{1-F\left(p_{0}\right)}-y .
$$

Similarly, at $t=p_{0} / \bar{y}, \tilde{y}_{s}\left(p_{0} / \bar{y}\right)=0$ and therefore, given $\lim _{y \rightarrow 0} f(y)=0$, $\left[d \tilde{y}_{s} / d t\right]_{t=p_{0} / \bar{y}}=-\bar{y}^{2} /\left(\bar{y}-p_{0}\right)$. Hence,

$$
\left.\frac{d u}{d t}\right|_{t=p_{0} / \bar{y}}=\bar{y}-y
$$

Claim (1).For all $y \geq \bar{y} /\left[1-F\left(p_{0}\right)\right],(8)$ and $d \tilde{y}_{s} / d t<0$ for all $t$ imply $d u(t ; y, 1) / d t<0$ on $\left(0, p_{0} / \bar{y}\right)$. Setting $y^{h} \equiv \bar{y} /\left[1-F\left(p_{0}\right)\right]$ completes the argument.

Claim (2). Let $y \in\left[\bar{y}, y^{h}\right)$. Then (8) implies $[d u(t ; y, 1) / d t]_{t=0}>0$, implying all individuals with incomes in this interval prefer some strictly positive tax rate to $t=0$. Moreover, (9) implies $[d u(t ; y, 1) / d t]_{t=p_{0} / \bar{y}}<0$ for all $y>\bar{y}$ and $[d u(t ; \bar{y}, 1) / d t]_{t=p_{0} / \bar{y}}=0$. Under the maintained assumption on the sign of $\sigma^{\prime \prime}(t)$, there can exist at most two stationary points of $u(t ; y, 1)$ on the interval $\left[0, p_{0} / \bar{y}\right]$. Claim $(2)$ now follows easily from a routine comparative static argument..

Claims (3) and (4). Let $y<\bar{y}$. Then (8) and (9), respectively, imply $[d u(t ; y, 1) / d t]_{t=0}>0$ and $[d u(t ; y, 1) / d t]_{t=p_{0} / \bar{y}}>0$. Further, (8), (9) and $d \tilde{y}_{s} / d t<0$ imply $d u(t ; y, 1) / d t>0$ on $\left(0, p_{0} / \bar{y}\right)$ for $y$ sufficiently small. Therefore, by $u(t ; y, 1)$ continuous in $y,[d u(t ; \bar{y}, 1) / d t]_{t=p_{0} / \bar{y}}=0$.and $u(0 ; \bar{y}, 1)=$ 
$u\left(p_{0} / \bar{y} ; \bar{y}, 1\right)$, there exists some $y^{l} \in(0, \bar{y})$ such that $\arg \max _{t} u\left(t ; y^{l}, 1\right)=$ $\left\{t\left(y^{l}\right), p_{0} / \bar{y}\right\}$ for some $t\left(y^{l}\right) \in\left(0, p_{0} / \bar{y}\right)$. Doing the comparative statics and invoking continuity once again completes the argument for Claims (3) and (4).

Claims (5) and (6). By Claim (4) and $d u(t ; 0,1) / d t>0$ on $\left(0, p_{0} / \bar{y}\right)$, Claim (4) and continuity of $u(t ; y, 1)$ in $y$ yield Claims (5) and (6).

Claim (7). For any $y \geq \tilde{y}_{s}(t), u^{*}(t ; y)=u(t ; y, 1)=y(1-t)+v-p(t) \geq v$; hence,

$$
u(t ; y, 1)-u(t ; y, 0)=v-p(t)>0 .
$$

By $\sigma^{\prime}(t)>0, p^{\prime}(t)<0$ on $\left[0, p_{0} / \bar{y}\right]$. So for any $t^{\prime} \in\left(t, p_{0} / \bar{y}\right)$,

$$
u\left(t^{\prime} ; y, 1\right)-u\left(t^{\prime} ; y, 0\right)=v-p\left(t^{\prime}\right)>v-p(t)>0 .
$$

This completes the proof.

The following lemma is useful for proving Proposition 2.

Lemma 2 For all $y \in\left(y^{l}, \bar{y}\right)$, let $s(y)=\left[\max t \in\left[0, p_{0} / \bar{y}\right): u(t ; y, 1)=\right.$ $\left.u\left(p_{0} / \bar{y} ; y, 1\right)\right]$. Then $s(y)$ is a well-defined and strictly increasing function of $y$ on $\left(y^{l}, \bar{y}\right)$. Moreover, $\lim _{y \rightarrow \bar{y}} s(y)=p_{0} / \bar{y}$.

\section{Proof of Lemma 2.}

Let $y \in\left(y^{l}, \bar{y}\right)$ and let $r(y) \in\left(t(y), p_{0} / \bar{y}\right)$ be the (unique) tax-rate minimizing $u(t ; y, 1)$ on $\left(t(y), p_{0} / \bar{y}\right)$; that $r(y)$ is well-defined for all $y \in\left(y^{l}, \bar{y}\right)$ follows from Lemma $1(3)$. Since $d u(t ; y, 1) / d t<0$ for all $t \in(t(y), r(y))$ and $y \in\left(y^{l}, \bar{y}\right)$, the tax-rate $s(y)$ is uniquely defined with $s(y) \in(t(y), r(y))$. And. by $u(t ; y, 1)$ differentiable in $y, s(y)$ is differentiable on its domain. By definition,

$$
u(s(y) ; y, 1)-u\left(p_{0} / \bar{y} ; y, 1\right) \equiv 0 .
$$

Substituting for $u(\cdot ; y, 1)$ and noting $\sigma\left(p_{0} / \bar{y}\right)=p_{0}$, the identity is equivalently

$$
[\sigma(s(y))-s(y) y]-p_{0}\left[1-\frac{y}{\bar{y}}\right] \equiv 0 .
$$

Differentiating through,

$$
s^{\prime}(y)\left[\sigma^{\prime}(s(y))-y\right]+\left[\frac{p_{0}}{\bar{y}}-s(y)\right] \equiv 0 .
$$


Now $d u(s(y) ; y, 1) / d t=\left[\sigma^{\prime}(s(y))-y\right]<0$ and $\left[p_{0} / \bar{y}-s(y)\right]>0$; therefore $s^{\prime}(y)>0$ as required. Finally, given $s^{\prime}(y)>0$, the limit claim follows from differentiability of $s(y)$ and Lemma 1(2) applied to $y=\bar{y}$.

\section{Proof of Proposition 2.}

Assume first that $t(\hat{y})<p_{0} / \bar{y}$. By Lemma 1, therefore, $\hat{y}>y^{l}$.

(Necessity)(2.1) Suppose $t>t(\hat{y})$. Then by Lemma 1, Lemma 2 and definition of $u^{*}(t ; y)=u(t ; y, 0)$, all individuals with incomes $y \geq \hat{y}$ strictly prefer $t(\hat{y})$ to $t$. By continuity of $F$, therefore, there exists a strict majority in favour of $t(\hat{y})$ against $t$, so $t$ cannot be a majority winner. Suppose $t=t(\hat{y})<$ $p_{0} / \bar{y}$; then $\tilde{y}_{s}(t)>0$ and $u^{*}(t ; y)=u(t ; y, 0)$ for all $y<\tilde{y}_{s}(t)$. Therefore, for $\varepsilon>0$ and sufficiently small, Lemmas 1 and 2 imply

$$
\forall y \in\left[0, \tilde{y}_{s}(t)\right) \cup(\hat{y}, \infty), u^{*}(t(\hat{y}) ; y)<u^{*}(t(\hat{y})-\varepsilon ; y) .
$$

By continuity of $F$, the interval $\left[0, \tilde{y}_{s}(t)\right)$ has strictly positive measure. Therefore,

$$
\int_{0}^{\tilde{y}_{s}(t)} d F(y)+\int_{\hat{y}}^{\infty} d F(y)>\frac{1}{2}
$$

and $t(\hat{y})$ canot be a majority winner. Now consider $t<\tilde{y}_{s}^{-1}(\hat{y})$. Then no individuals with income $y \leq \hat{y}$ can afford to buy the good so $u^{*}(t ; y)<$ $u(t ; y, 1)$ for all $y \leq \hat{y}$ and there exists a strict majority in favour of zero taxes against $t$. Thus $t \in\left[\tilde{y}_{s}^{-1}(\hat{y}), t(\hat{y})\right)$ is a necessary condition for any majority winner.

(Necessity)(2.2) Let $t \in\left[\tilde{y}_{s}^{-1}(\hat{y}), t(\hat{y})\right)$. By the observation following Lemma 1 that $t^{-1}(t)>\tilde{y}_{s}(t), u^{*}(t-\varepsilon ; y)>u^{*}(t ; y)$ for all $y>t^{-1}(t)$ and some small $\varepsilon>0$. And, by definition of $\tilde{y}_{s}(t), u^{*}\left(t^{\prime} ; y\right)>u^{*}(t ; y)$ for all $t^{\prime}<t$ and all $y<\tilde{y}_{s}(t)$. Consequently, $t$ can be a majority winner against $t-\varepsilon$, $\varepsilon>0$, only if $\int_{\tilde{y}_{s}(t)}^{t^{-1}(t)} d F(y) \geq 1 / 2$. Suppose $\int_{\tilde{y}_{s}(t)}^{t^{-1}(t)} d F(y)>1 / 2$; then, for small $\varepsilon>0, u^{*}(t+\varepsilon ; y)>u^{*}(t ; y)$ for all $y \in\left(\tilde{y}_{s}(t+\varepsilon), t^{-1}(t+\varepsilon)\right)$. By $(5)$ and Lemma $1, \tilde{y}_{s}(t+\varepsilon)<\tilde{y}_{s}(t)$ and $t^{-1}(t+\varepsilon)<t^{-1}(t)$. Hence, for $\varepsilon$ sufficiently small, $\int_{\tilde{y}_{s}(t+\varepsilon)}^{\omega(t, t+\varepsilon)} d F(y)>1 / 2$ in which case $t+\varepsilon$ is strictly majority preferred to $t$.

(Necessity)(2.3) If $p_{0} / \bar{y} \in P\left(\tau_{s}\right)$ then $u^{*}\left(p_{0} / \bar{y} ; y\right)>u^{*}\left(\tau_{s} ; y\right)$ for all $y \leq \hat{y}$ so $p_{0} / \bar{y}$ is strictly preferred by a majority to $\tau_{s}$.

(Necessity) (2.4) Let $\tau_{s}$ satisfy conditions (2.1), (2.2) and (2.3). Suppose there is some $t \in P\left(\tau_{s}\right)$ for which $\int_{\tilde{y}_{s}(t)}^{\omega\left(\tau_{s}, t\right)} d F(y)>\frac{1}{2}$. Then $t$ is strictly 
majority preferred to $\tau_{s}$ : by Lemma 1 and $t \in P\left(\tau_{s}\right), u^{*}\left(\tau_{s} ; y\right)>u^{*}(t ; y)$ only if $y \notin\left[\tilde{y}_{s}(t), \omega\left(\tau_{s}, t\right)\right)$. This completes the proof of necessity.

(Sufficiency) Assume conditions (2.1), (2.2), (2.3) and (2.4) hold at $\tau_{s}$. Let $t<\tau_{s}$. By Lemma 1 and $\hat{y}<y^{l}$, an individual strictly prefers $\tau_{s}$ to $t$ if and only if $y \in\left[\tilde{y}_{s}\left(\tau_{s}\right), \omega\left(t, \tau_{s}\right)\right)$. But $\left[\tilde{y}_{s}\left(\tau_{s}\right), \omega\left(t, \tau_{s}\right)\right) \supset\left(\tilde{y}_{s}\left(\tau_{s}\right), t^{-1}\left(\tau_{s}\right)\right)$ in which case conditions (2.1) and (2.2) imply

$$
\int_{Y\left(t, \tau_{s}\right)} d F(y)<\frac{1}{2}
$$

so $\tau_{s}$ is strictly majority preferred to $t$. Now consider $t>\tau_{s}$. If $t \notin P\left(\tau_{s}\right)$, then Lemma 1 implies a strict majority strictly prefers $\tau_{s}$ to $t$. If $t \in P\left(\tau_{s}\right)$, then condition (2.3) says $t<p_{0} / \bar{y}$ so, by Lemma $1, P\left(\tau_{s}\right)$ is an interval $\left(\tau_{s}, b\right)$ for some $b \in\left(t(\hat{y}), p_{0} / \bar{y}\right)$; condition (2.4) then directly insures that $\tau_{s}$ is majority preferred to $t$.

(Uniqueness) The first part of the preceding sufficiency argument implies that if both $t$ and $t^{\prime}$ satisfy conditions (2.1) and (2.2) with $t<t^{\prime}$, then condition (2.4) cannot obtain at $t$. In particular, at most the maximal tax-rate satisfying both conditions (2.1) and (2.2) can possibly be a majority winner. This proves uniqueness and completes the argument for the proposition.

\section{Proof of Proposition 3.}

Since $\hat{y}>0, u^{*}\left(\tau_{s} ; \hat{y}\right)=u^{*}\left(\tau_{r} ; \hat{y}\right)$ under either the subsidy or the redistribution policy. Consequently, if $\tau_{s}=\tau_{r}=p_{0} / \bar{y}$ then clearly a majority is indifferent between the two. On the other hand, if $\tau_{s}<\tau_{r}$ then $\hat{y}>y^{l}$ and, by condition (2.3) of Proposition $2, u^{*}\left(\tau_{s} ; \hat{y}\right)>u^{*}\left(p_{0} / \bar{y} ; \hat{y}\right)$ necessarily; by Lemma 1 therefore, $u^{*}\left(\tau_{s} ; y\right)>u^{*}\left(p_{0} / \bar{y} ; y\right)$ for all $y \geq \hat{y}$ and a strict majority prefers $\tau_{s}$ to $\tau_{r}$.

Suppose a small proportion $\phi>0$ of the population values the good at zero and so never consume it $\left(v^{\prime}=0\right)$. Then the marginal consumer at tax-rate $t$ is implicitly defined by

$$
\tilde{y}(t)(1-t)+\frac{t \bar{y}}{(1-\phi)(1-F(\tilde{y}(t))} \equiv p_{0}
$$

and the tax-rate at which $\tilde{y}(t)=0$ is $t=p(1-\phi) / \bar{y}$. Implicitly differentiating and collecting terms yields

$$
\frac{d \tilde{y}}{d t}=\frac{[1-F(\tilde{y})][(1-F(\tilde{y}))(1-\phi) \tilde{y}-\bar{y}]}{(1-t)(1-F(\tilde{y}))^{2}(1-\phi)+t \bar{y} f(\tilde{y})} .
$$


Using these expressions, Lemma 1 (mutatis mutandis) continues to hold; the cutpoints characterizing the various sorts of induced preference over taxrates, however, shift upward. For example, the set of incomes having local but not global maxima at $t=p_{0}(1-\phi) / \bar{y}$ becomes the interval $\left(y^{l^{\prime}}, \bar{y} /(1-\phi)\right)$, where $y^{l^{\prime}}>y^{l}$. It follows that for $\phi$ small, continuity implies the existence result goes through as described in the text.

Finally, to check the comparative static on the majority winner, note

$$
\left.\frac{d u}{d t}\right|_{t<(1-\phi) p_{0} / \bar{y}}=[\tilde{y}-y]-\frac{d \tilde{y}}{d t}(1-t) .
$$

Consider $y$ such that $y$ 's most preferred tax-rate, say $t(y ; \phi)$, is interior. Then the second-order condition holds and $\operatorname{sgn}[d t(y ; \phi) / d \phi]=-\operatorname{sgn}\left[\partial^{2} u / \partial t \partial \phi\right]$. Doing the calculus,

$\frac{\partial^{2} u}{\partial t \partial \phi} \propto(1-F(\tilde{y}))[(1-F(\tilde{y}))(1-\phi) \tilde{y}-\bar{y}]-\left[(1-t)(1-F(\tilde{y}))^{2}(1-\phi)+t \bar{y} f(\tilde{y})\right]<0$.

Hence, $d t(y ; \phi) / d \phi>0$ and the claim follows.

\section{Appendix B: Computations}

Recall the distribution of income,

$$
F(y)=\left\{\begin{array}{cll}
\frac{y}{6} & \text { if } & 0 \leq y<1 \\
\frac{1}{6}+\frac{2(y-1)}{3} & \text { if } & 1 \leq y<2 \\
\frac{5}{6}+\frac{(y-2)}{42} & \text { if } & 2 \leq y \leq 9
\end{array}\right.
$$

So mean and median incomes are, respectively, $\bar{y}=2$ and $\hat{y}=3 / 2$. Because $f(0)=1 / 6$, induced preferences over tax-rates for $y \geq 6 / 5$ are strictly quasiconcave; in particular, the median income person, and therefore a strict majority, has such induced preferences. If $p_{0} \in[1,2)$ then $\tilde{y}_{s}(t) \leq 2$ for all $t$ and (3) yields

$$
t\left(\tilde{y}_{s}\right)=\left\{\begin{array}{lll}
\frac{6 p_{0}-\left(p_{0}+6\right) \tilde{y}_{s}+\tilde{y}_{s}^{2}}{12-6 \tilde{y}_{s}+\tilde{y}_{s}^{2}} & \text { if } & 0 \leq \tilde{y}_{s} \leq 1 \\
\frac{9 p_{0}-\left(4 p_{0}+9\right) \tilde{y}_{s}+4 \tilde{y}_{s}^{2}}{12-9 \tilde{y}_{s}+4 \tilde{y}_{s}^{2}} & \text { if } & 1<\tilde{y}_{s} \leq 2
\end{array} .\right.
$$


The subsidy is $\sigma\left(t\left(\tilde{y}_{s}\right)\right)=2 t\left(\tilde{y}_{s}\right) /\left[1-F\left(\tilde{y}_{s}\right)\right]$ and, for $p_{0} \in[1,2)$, can be checked to satisfy the maintained assumption that $\sigma^{\prime \prime}(t)$ can change sign at most once, from minus to plus, on the interval $\left[0, p_{0} / \bar{y}\right]$. The median individual's utility is given by $u(t ; \hat{y}, 1)=3 / 2+v+U\left(\tilde{y}_{s}\right)$, where

$$
U\left(\tilde{y}_{s}\right)=t\left(\tilde{y}_{s}\right)\left[\frac{2}{1-F\left(\tilde{y}_{s}\right)}-\frac{3}{2}\right] .
$$

Detailed computations are given only for the second row of Table 1 ; the remaining cases, $p_{0} \in\{5 / 3,1\}$, follow in the same way.

Suppose the unit price of $v$ is $p_{0}=4 / 3$. Then the median income individual can consume $v$ whether or not there are any subsidies, yet the median's most preferred tax-rate is $t(\hat{y})=0.58$ implying $\tilde{y}_{s}(t(\hat{y}))=0.29$. Consequently, there exists a slightly lower tax-rate to $t(\hat{y})$ that is strictly preferred to $t(\hat{y})$ by a majority from those with incomes $y \in(1.5,9] \cup[0,0.29)$. On the other hand, the tax-rate $\tau_{s}=0.54<t(\hat{y})$ is most preferred by the individual with income $t^{-1}(0.54)=1.59$ and this rate induces consumption by all $y \geq 0.38$. Moreover,

$$
F(1.59)-F(0.38)=0.50
$$

and

$$
u^{*}\left(\tau_{s} ; \hat{y}\right)-u^{*}\left(p_{0} / \bar{y} ; \hat{y}\right)=0.35-0.33=0.02 \text {. }
$$

Thus conditions (2.1), (2.2) and (2.3) are satisfied at $\tau_{s}=0.54$; it remains to check condition (2.4). First, it is straightforward to confirm that no taxrate $t \in(0.54,0.58)$ satisfies condition $(2.2)$ and that the set of alternatives strictly preferred by the median voter to $\tau_{s}, P\left(\tau_{s}\right)$, is the interval $(0.54,0.62)$. So for $t \in P\left(\tau_{s}\right), \omega\left(\tau_{s}, t\right) \in(1.5,1.59)$ and $\tilde{y}_{s}(t) \in(0.2,0.38)$. Now, because condition (2.2) holds at $\tau_{s}$, a sufficient condition for condition (2.4) to hold is that

$$
\frac{\left[d \tilde{y}_{s}(t) / d t\right]}{\left[d \omega\left(\tau_{s}, t\right) / d t\right]} \leq \frac{f\left(\omega\left(\tau_{s}, t\right)\right)}{f\left(\tilde{y}_{s}(t)\right)}
$$

on $P\left(\tau_{s}\right)$ (see fn.7). From (10), both $\tilde{y}_{s}$ and $\omega\left(\tau_{s}, t\right)=\left[\sigma(t)-\sigma\left(\tau_{s}\right)\right] /\left[t-\tau_{s}\right]$ are approximately linear over $P\left(\tau_{s}\right)$; hence

$$
\frac{d \tilde{y}_{s}(t)}{d t} \approx \frac{0.2-0.38}{0.62-0.54}=-9 / 4
$$


and

$$
\frac{d \omega\left(\tau_{s}, t\right)}{d t} \approx \frac{1.5-1.59}{0.62-0.54}=-9 / 8
$$

implying the left side of (11) (approximately) equals 2 . However, since $2>$ $\omega\left(\tau_{s}, t\right)>1>\tilde{y}_{s}(t)$ for all $t \in P\left(\tau_{s}\right)$, the right side of (11) equals 4 . Therefore condition (2.4) holds and $\tau_{s}=0.54$ is a majority winner under the subsidy policy. And clearly, the subsidy policy here is majority preferred to the redistribution policy, under which the majority winner is $\tau_{r}=p_{0} / \bar{y}=0.67$. 
Acknowledgement. This version of the paper has benefitted greatly from the comments of an anonymous referee, to whom I am grateful. I am also grateful to the John D. and Catherine T. MacArthur Foundation for financial support through the Social Interactions and Economic Inequality Network. I retain all responsibility for the content of the paper.

\section{References}

[1] Besley, T. and S. Coate. 1991. Public provision of private goods and the redistribution of income. American Economic Review, 81:979-984.

[2] Fernandez, R. and R. Rogerson. 1995. On the political economy of education subsidies. Review of Economic Studies, 62:249-262.

[3] Gans, J. and M. Smart. 1996. Majority voting with single-crossing preferences. Journal of Public Economics, 59:219-237.

[4] Hall, Sir Peter. 1999. Interview. Cam, Michaelmas Issue. Cambridge University Press.

[5] Rothstein, P. 1990. Order-restricted preferences and majority rule. Social Choice and Welfare, 7:331-342. 
Figure 1: Redistribution policy 
Figure 2: Maximal utility, $y \in\left(y^{l}, \bar{y}\right)$ 
Figure 3: Consumption subsidy policy 
Figure 4: Majority winner under subsidy policy 